University of New Orleans

ScholarWorks@UNO

4-1978

\title{
Simulation of mechanical rotation by optical rotation: Application to the design of a new Fourier photopolarimeter
}

R. M.A. Azzam

University of New Orleans, razzam@uno.edu

Follow this and additional works at: https://scholarworks.uno.edu/ee_facpubs

Part of the Electrical and Electronics Commons, and the Physics Commons

\section{Recommended Citation}

R. M. A. Azzam, "Simulation of mechanical rotation by optical rotation: Application to the design of a new Fourier photopolarimeter," J. Opt. Soc. Am. 68, 518-521 (1978)

This Article is brought to you for free and open access by the Department of Electrical Engineering at ScholarWorks@UNO. It has been accepted for inclusion in Electrical Engineering Faculty Publications by an authorized administrator of ScholarWorks@UNO. For more information, please contact scholarworks@uno.edu. 
sian Jones matrix with elements $T_{12}=T_{21}=0, T_{11}=\tan \psi e^{j \Delta}$, and $T_{22}=1$. ( $\psi$ and $\Delta$ are the usual ellipsometric parameters.) If we substitute these elements in Eqs. (A2), and assume linearly polarized incident light so that $\chi_{i}=\tan \theta_{i}$, we obtain from Eq. (A1) the following ARF:

$$
\tan 2 \theta_{o}=\frac{2 \tan \theta_{i} \tan \psi \cos \Delta}{\tan ^{2} \psi-\tan ^{2} \theta_{i}},
$$

which agrees with Eq. (3) of Ref. 1, as expected.

If, in the above example, we try to determine the ARF by first finding the equivalent circular Jones matrix and then using any of the expressions of the ARF in Secs. II or III, we discover that this procedure is indeed considerably more complicated.

Noted added in proof. A recent article ${ }^{14}$ provides a significant additional reference for this paper.

1J. Monin and G.-A. Boutry, "Conception, réalisation et fonctionnement d'un nouvel ellipsomètre," Nouv. Rev. Opt. 4, 159-169 (1973); and Refs. (19) and (20) listed therein.

${ }^{2}$ S. C. Som and C. Chowdhury, "New ellipsometric method for the determination of the optical constants of thin films and surfaces,"
J. Opt. Soc. Am. 62, 10-15 (1972).

3Such an extension is a special case of generalized ellipsometry (see, e.g., R. M. A. Azzam and N. M. Bashara, "Applications of generalized ellipsometry to anisotropic crystals," J. Opt. Soc. Am. 64, 128-133 (1974)).

${ }^{4}$ For definiteness, we assume that $\theta_{i}$ and $\theta_{o}$ are measured from the plane of the incident and outgoing beams.

${ }^{5}$ R. M. A. Azzam and N. M. Bashara, Ellipsometry and Polarized Light (North-Holland, Amsterdam, 1977), Sec. 2.2.5.

${ }^{6}$ Reference 5, Sec. 1.7.2.

${ }^{7}$ Reference 5, Sec. 2.3.

${ }^{8}$ The ensuing analysis can easily be adapted to the general case of any (but fixed) input ellipticity $\left(\epsilon_{i} \neq 0\right)$.

${ }^{9}$ R. M. A. Azzam, "Hybrid null-photometric ellipsometer using sinusoidal optical rotation," Optik 48, 279-288 (1977).

${ }^{10}$ A. V. S. S. S. R. Sarma, "New experimental methods for determining the optical parameters of elliptic retarders," J. Phys. D: Appl. Phys. 10, 2019-2030 (1977).

11F. El-Hosseiny, "Methods for determining the optical parameters of elliptic retarders," J. Opt. Soc. Am. 65, 1279-1282 (1975).

${ }^{12}$ Reference 5 , p. 488.

${ }^{13}$ R. C. Jones, "A new calculus for the treatment of optical systems. VII, Properties of the N-matrices," J. Opt. Soc. Am. 38, 6'71-685 (1948).

${ }^{14} \mathrm{~S}$. I. Idnurm, "Method of determing the parameters of inhomogeneous anisotropic media using a photoelectric polariscope," Opt. Spectrosc. 42, 210-212 (1977).

\title{
Simulation of mechanical rotation by optical rotation: Application to the design of a new Fourier photopolarimeter
}

\author{
R. M. A. Azzam \\ Hematology Division and Department of Internal Medicine, University of Nebraska Medical Center, Omaha, Nebraska 68105 \\ and Electrical Materials Laboratory, College of Engineering, University of Nebraska, Lincoln. Nebraska 68588 \\ (Received 28 January 1977)

\begin{abstract}
The mechanical rotation of an optical element around the axis of a beam of polarized light can be easily simulated by using the phenomenon of optical rotation. Because optical rotation can be magnetically or electrically induced, virtually any kind of mechanical rotation can be mimicked. This interesting principle is applied to the design of a new Fourier photopolarimeter that uses an oscillating-azimuth retarder (OAR). The OAR consists of a quarter-wave plate surrounded by two ac-excited Faraday cells that produce equal and opposite sinusoidal optical rotations. Analysis of the operation of this polarimeter of no moving parts proves its ability to measure all four Stokes parameters of incident partially polarized radiation.
\end{abstract}

\section{INTRODUCTION}

In this paper we show that the mechanical rotation of an optical element around the axis of a beam of polarized light can be easily simulated by using the phenomenon of optical rotation. Because optical rotation can be induced and controlled by magnetic and electric fields (e.g., in Faraday and liquid-crystal cells), it is possible, in principle, to simulate, any type of mechanical rotation by tailoring the required electric or magnetic input. After giving the formal proof of this interesting concept, we apply it to the design of a new (automatic) Fourier photopolarimeter which is capable of measuring the four Stokes parameters of a partially polarized light beam. Applications other than in polarimetry are anticipated, e.g., in the up/down frequency shifter of Sommargren ${ }^{1}$ that requires a synchronously rotating half-wave plate.

\section{CONTROL OF THE AZIMUTH OF AN OPTICAL ELEMENT BY OPTICAL ROTATION}

In Fig. 1, let $\mathrm{T}_{\mathrm{E}}^{x, y}$ be the (Mueller or Jones) matrix of an optical element $E$ with respect to a transverse coordinate system $x y$ fixed to the element. We assume that $x y$ is rotated around the beam axis with respect to another reference coordinate system $x^{\prime} y^{\prime}$ by the azimuth

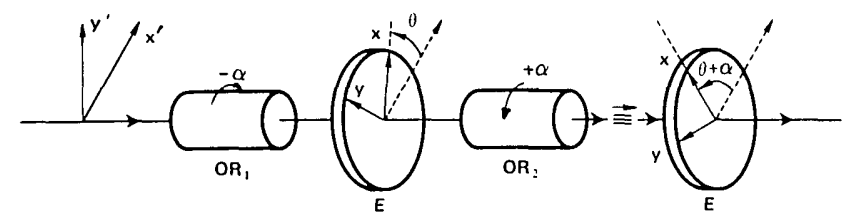

FIG. 1. Optical element $\mathbf{E}$ of azimuth $\theta$ which is surrounded by two optical rotators $\mathrm{OR}_{1}$ and $\mathrm{OR}_{2}$ with equal and opposite rotations $+\alpha$ and $-\alpha$ is equivalent to itself in a new azimuth position $\theta+\alpha$. 
angle $\theta$. The matrix of $\mathrm{E}$ in the $x^{\prime} y^{\prime}$ coordinate system is then given by

$$
\mathbf{T}_{\mathrm{E}}^{x^{\prime}, y^{\prime}}=\mathbf{R}(-\theta) \mathbf{T}_{\mathrm{E}}^{x, y} \mathbf{R}(\theta),
$$

where $R(\theta)$ is the coordinate rotation matrix. In Fig. 1, $\mathrm{OR}_{1}$ and $\mathrm{OR}_{2}$ represent two optical rotators placed one immediately before and the other immediately after the element $E . O_{1}$ and $O R_{2}$ give equal and opposite optical rotations $-\alpha$ and $+\alpha$, respectively. The matrix of the element $E$ and the surrounding optical rotators $O_{1}$ and $\mathrm{OR}_{2}$ is given by

$$
\mathrm{T}_{\mathrm{OR}_{1}+\mathrm{E}+\mathrm{OR}_{2}}^{x^{\prime}, y^{\prime}}=\mathbf{R}(-\alpha) \mathbf{T}_{\mathrm{E}}^{x^{\prime}, y^{\prime}} \mathbf{R}(\alpha),
$$

where $\mathbf{R}(\alpha)$ and $\mathbf{R}(-\alpha)$ are the optical rotation matrices of $\mathrm{OR}_{1}$ and $\mathrm{OR}_{2}$, respectively. Upon substituting Eq.

(1) into Eq. (2), we get

$$
\begin{aligned}
\mathbf{T}_{\mathrm{OR}_{1}+\mathrm{E}+\mathrm{OR}_{2}}^{x^{\prime}, y^{\prime}} & =\mathbf{R}(-\alpha) \mathbf{R}(-\theta) \mathbf{T}_{\mathrm{E}}^{x, y} \mathbf{R}(\theta) \mathbf{R}(\alpha) \\
& =\mathbf{R}[-(\theta+\alpha)] \mathbf{T}_{\mathrm{E}}^{x, y} \mathbf{R}[+(\theta+\alpha)],
\end{aligned}
$$

where we have used the following property of the rotation matrix:

$$
\mathbf{R}(x) \mathbf{R}(y)=\mathbf{R}(x+y) .
$$

It should be observed that while the coordinate and optical rotations are similarly represented mathematically, they are physically distinguishable.

Equation (3) shows that the element $E$ and the surrounding optical rotators $\mathrm{OR}_{1}$ and $\mathrm{OR}_{2}$ are equivalent to the element $\mathrm{E}$ alone in the new azimuth position $\theta+\alpha$, where $\alpha$ is the magnitude of the optical rotation introduced by $O R_{1}$ and $O R_{2}$. This equivalence is schematically illustrated in Fig. 1.

By magnetically or electrically controlling $\alpha$, we can simulate any mechanical rotation desired. For example, a periodic saw-tooth optical rotation

$$
\begin{aligned}
& \alpha=\omega(t-n T), \quad\left(n-\frac{1}{2}\right) T \leqslant t \leqslant\left(n+\frac{1}{2}\right) T, \\
& n=0, \pm 1, \pm 2, \ldots, \quad \omega T=2 \pi
\end{aligned}
$$

simulates synchronous mechanical rotation of the ele-

(a)

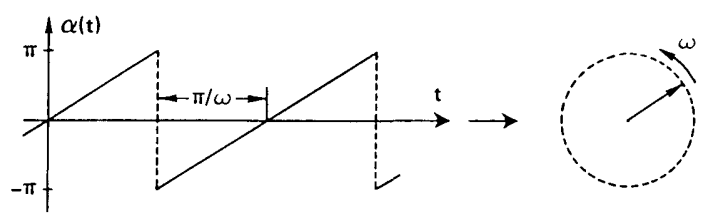

(b)

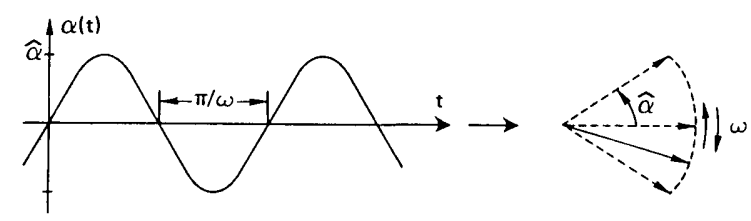

FIG. 2. (a) If the optical rotation $\alpha$ (in Fig. 1) varies as a saw-tooth function of time, the equivalent element $\mathrm{E}$ will be synchronously rotating. (b) If the optical rotation $\alpha$ varies sinusoidally with time, the equivalent element $\mathrm{E}$ will execute a pendulumlike oscillation. ment $\mathrm{E}$ at constant angular speed $\omega$. A sinusoidal optical rotation

$$
\alpha=\hat{\alpha} \sin \omega t
$$

is simpler to achieve and simulates pendulumlike mechanical oscillation of the element $\mathrm{E}$. These examples are illustrated in Fig. 2.

\section{APPLICATION TO THE DESIGN OF A NEW FOURIER PHOTOPOLARIMETER}

The state of polarization of a light beam (described, for example, by the four Stokes parameters) is often measured by Fourier analysis of the light-intensity variations detected after the beam has passed through a train of optical components one or more of which is modulated periodically with time. A large variety of these "Fourier photopolarimeters" has been described with different sequences of optical elements and different modulation schemes. ${ }^{2-6}$

Two modulation techniques have been widely employed: (i) azimuth modulation, achieved by the continuous synchronous mechanical rotation of one or more optical elements (e.g., a linear polarizer and/or linear retarder ${ }^{5}$ ), and (ii) phase (birefringence) modulation, obtained by oscillating the relative phase shift of an electro-optical ${ }^{7}$ or piezo-optical ${ }^{8}$ retarder.

By contrast, modulated optical rotation has remained largely unexploited in the design of Fourier photopolarimeters. An exception is the oscillating-analyzer ellipsometer ${ }^{9}$ (OAE) that consists of an ac-driven Faraday cell followed by a fixed linear analyzer. The sinusoidal magnetic optical rotation induced by the ac Faraday cell effectively oscillates the azimuth of the linear analyzer. The need for mechanical rotation of the analyzer is therefore averted and the advantages of an ellipsometer with stationary optics (high speed and easy alignment) are achieved. The OAE, however, is only suited for the analysis of totally polarized and, in that, it is incapable of specifying the handedness of polarization.

In this section we introduce a new Fourier photopolarimeter that, like the OAE, uses sinusoidal optical rotation but, unlike the OAE, is capable of completely measuring the state of polarization (the Stokes vector) of partially (or totally) polarized light. The polarimeter, Fig. 3, consists of an oscillating-azimuth retarder (OAR) followed by a fixed linear analyzer A. Oscillation of the azimuth of a quarter-wave retarder (QWR) is nonmechanically realized by surrounding it by two sinusoidal optical rotators (ac-excited Faraday cells $\mathrm{FC}_{1}$ and $\mathrm{FC}_{2}$ ) that give equal and opposite rotations. From limited Fourier analysis of the output signal $i$ of the linear photodetector $\mathrm{D}$ (which receives the light flux leaving the analyzer), we can determine the Stokes parameters (the components of the Stokes vector S) of the light incident on the polarimeter. This is explained in the subsequent analysis.

Let the Stokes parameters of the light beam under measurement be $S_{0}, S_{1}, S_{2}, S_{3}$. After the passage of the light beam through a linear retarder of relative 


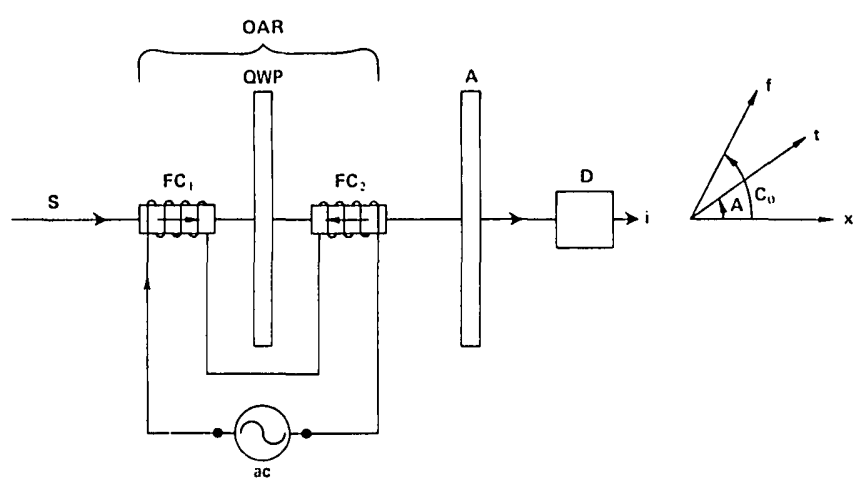

FIG. 3. New Fourier photopolarimeter using an oscillatingazimuth retarder (OAR). The OAR consists of a fixed quartersquare plate (QWP) surrounded by two identical ac-excited Faraday cells $\mathrm{FC}_{1}$ and $\mathrm{FC}_{2}$ that produce equal and opposite sinusoidal optical rotations. (Additonal dc excitation of the Faraday cells may be utilized to control the bias azimuth $C_{0}$.) $A$ is a fixed linear analyzer and $D$ is a linear photodetector. Fourier analysis of the output signal $i$ of $D$ gives the Stokes vector $S$ of the light incident on the polarimeter. The azimuthal orientations $\mathrm{A}$ of the transmission axis $t$ of the analyzer and $C_{0}$ of the fast axis $f$ of the QWP are indicated (right).

phase shift (retardance) $\delta$ and fast-axis azimuth $C$ and an ideal linear analyzer of transmission-axis azimuth $A$, the Stokes parameter $S_{0}^{\prime}$ (equal to the total intensity) of the emergent light is given by ${ }^{10}$

$$
\begin{aligned}
2 S_{0}^{\prime}= & S_{0}+S_{1}[\cos 2 C \cos (2 A-2 C)-\sin 2 C \sin (2 A-2 C) \cos \delta] \\
& +S_{2}[\sin 2 C \cos (2 A-2 C)+\cos 2 C \sin (2 A-2 C) \cos \delta] \\
& +S_{3}[\sin (2 A-2 C) \sin \delta] .
\end{aligned}
$$

The emergent light impinges on the linear photodetector $\mathrm{D}$ to give a signal $i$ proportional to $S_{0}^{\prime}$ (i. e. , $i=$ const - $S_{0}^{\prime}$ ). If we assume $\delta=\frac{1}{2} \pi$ (i. e. , a quarter-wave retarder) and $A=0$, we get from Eq. (7):

$$
k i=\left(S_{0}+\frac{1}{2} S_{1}\right)+\frac{1}{2} S_{1} \cos 4 C+\frac{1}{2} S_{2} \sin 4 C-S_{3} \sin 2 C,
$$

or

$$
k i=\beta_{0}+\beta_{1} \cos 4 C+\beta_{2} \sin 4 C+\beta_{3} \sin 2 C,
$$

where

$$
\beta_{0}=S_{0}+\frac{1}{2} S_{1}, \quad \beta_{1}=\frac{1}{2} S_{1}, \quad \beta_{2}=\frac{1}{2} S_{2}, \quad \beta_{3}=-S_{3},
$$

and $k$ is a proportionality constant. The choice $A=0$ does not reduce the generality of the discussion, as it implies that the Stokes parameters are defined with respect to a coordinate system coincident with the transmission and extinction axes of the analyzer. The Stokes parameters in a different coordinate system can be easily obtained by a coordinate transformation described by the $4 \times 4$ Mueller rotation matrix. Generalization to an arbitrary retarder phase angle $\delta$ is straightforward but the results are necessarily more complicated.

According to the discussion in Sec. II, the addition of the two ac optical rotators one on each side of the retarder effectively oscillates the retarder's azimuth, so that

$$
C=C_{0}+\hat{c} \sin \omega t
$$

where $C_{0}$ is a constant bias azimuth and $\hat{c}$ is the opticalrotation amplitude. If we substitute Eq. (11) into (9),

$$
\begin{aligned}
k \hat{i}_{3 \omega}= & \beta_{1}\left[-2 \sin 4 C_{0} J_{3}(4 \hat{c})\right]+\beta_{2}\left[2 \cos 4 C_{0} J_{3}(4 \hat{c})\right] \\
& +\beta_{3}\left[2 \cos 2 C_{0} J_{3}(2 \hat{c})\right] .
\end{aligned}
$$

Equations (13b)-(13d) can be put in matrix form as

$$
k \hat{\mathrm{i}}=\mathbf{C}_{\boldsymbol{\beta}},
$$

where

$$
\hat{\mathbf{i}}=\left(\begin{array}{c}
\hat{i}_{\omega} \\
\hat{i}_{2 \omega} \\
\hat{i}_{3 \omega}
\end{array}\right), \quad \boldsymbol{\beta}=\left(\begin{array}{c}
\beta_{1} \\
\beta_{2} \\
\beta_{3}
\end{array}\right)
$$

and

$$
\mathrm{C}=\left(\begin{array}{ccc}
-2 \sin 4 C_{0} J_{1}(4 \hat{c}) & 2 \cos 4 C_{0} J_{1}(4 \hat{c}) & 2 \cos 2 C_{0} J_{1}(2 \hat{c}) \\
2 \cos 4 C_{0} J_{2}(4 \hat{c}) & 2 \sin 4 C_{0} J_{2}(4 \hat{c}) & 2 \sin 2 C_{0} J_{2}(2 \hat{c}) \\
-2 \sin 4 C_{0} J_{3}(4 \hat{c}) & 2 \cos 4 C_{0} J_{3}(4 \hat{c}) & 2 \cos 2 C_{0} J_{3}(2 \hat{c})
\end{array}\right) .
$$

The solution of Eq. (14) for $\beta$ is

$$
\boldsymbol{\beta}=k \mathbf{C}^{-1} \hat{\mathrm{i}} \text {, }
$$

where $\mathrm{C}^{-1}$ is the inverse of the matrix $\mathrm{C}$ of $\mathrm{Eq}$. (15b).

Thus, by measuring the amplitudes of the fundamental, second, and third harmonics $\left(\hat{i}_{\omega}, \hat{i}_{2 \omega}, \hat{i}_{3 \omega}\right)$ of the signal $i$, and evaluating the inverse of the matrix $\mathbf{C}$ of Eq. (15b) for given values of $C_{0}$ and $\hat{c}$, we can use Eq. (16) to get $\beta \equiv\left(\beta_{1}, \beta_{2}, \beta_{3}\right)$ and determine $\beta_{0}$ subsequently from Eq. (13a). The Stokes parameters $S_{0}, S_{1}, S_{2}, S_{3}$ of the light beam under measurement are next obtained from $\beta_{0}, \beta_{1}, \beta_{2}, \beta_{3}$ using Eqs. (10).

The Stokes parameters are not absolutely determined unless the value of the constant multiplier $k$ is measured, e.g., using a reference beam of known intensity and polarization. However, we are often interested only in the information on polarization which is contained in the normalized Stokes parameters $S_{1} / S_{0}, S_{2} / S_{0}, S_{3} / S_{0}$. and make use of the Bessel-function expansions ${ }^{11}$

$$
\begin{aligned}
& \cos (x \sin \omega t)=J_{0}(x)+2 \sum_{n=1}^{\infty} J_{2 n}(x) \cos 2 n \omega t, \\
& \sin (x \sin \omega t)=2 \sum_{n=0}^{\infty} J_{2 n+1}(x) \sin [(2 n+1) \omega t],
\end{aligned}
$$

we obtain the following Fourier amplitudes of the signal $i$ :

$$
\begin{aligned}
k i_{d c}= & \beta_{0}+\beta_{1}\left[\cos 4 C_{0} J_{0}(4 \hat{c})\right]+\beta_{2}\left[\sin 4 C_{0} J_{0}(4 \hat{c})\right] \\
& +\beta_{3}\left[\sin 2 C_{0} J_{0}(2 \hat{c})\right], \\
k \hat{i_{\omega}}= & \beta_{1}\left[-2 \sin 4 C_{0} J_{1}(4 \hat{c})\right]+\beta_{2}\left[2 \cos 4 C_{0} J_{1}(4 \hat{c})\right] \\
& +\beta_{3}\left[2 \cos 2 C_{0} J_{1}(2 \hat{c})\right], \\
k \hat{i}_{2 \omega}= & \beta_{1}\left[2 \cos 4 C_{0} J_{2}(4 \hat{c})\right]+\beta_{2}\left[2 \sin 4 C_{0} J_{2}(4 \hat{c})\right] \\
& +\beta_{3}\left[2 \sin 2 C_{0} J_{2}(2 \hat{c})\right],
\end{aligned}
$$

The bias azimuth $C_{0}$ and optical-rotation amplitude $\hat{c}$ are two design parameters that can be specified at will to achieve some advantage. For example, if we choose $C_{0}=0$ or $\pm \frac{1}{2} \pi$ (i. e., when the fast axis of the retarder is parallel to the transmission axis or the extinction axis of the analyzer), the matrix $\mathbf{C}$ simplifies to 


$$
\left.\mathrm{C}\right|_{C_{0}=0, \pm \pi / 2}=\left(\begin{array}{ccc}
0 & 2 J_{1}(4 \hat{c}) & \pm 2 J_{1}(2 \hat{c}) \\
2 J_{2}(4 \hat{c}) & 0 & 0 \\
0 & 2 J_{3}(4 \hat{c}) & \pm 2 J_{3}(2 \hat{c})
\end{array}\right),
$$

where the upper and lower signis correspond to $C_{0}=0$ and $C_{0}= \pm \frac{1}{2} \pi$, respectively. In this case, Eq. (13c) gives $\beta_{2}$, and Eqs. (13b) and (13d) are readily solvable for $\beta_{1}$ and $\beta_{3}$. Of course, the same result could be obtained if the matrix of Eq. (17) is inverted and Eq. (16) is used.

Not all choices of $C_{0}$ and $\hat{c}$ permit the determination of all $\beta$ 's, i. e., all the Stokes parameters. This happens, for example, when $C_{0}= \pm \frac{1}{4} \pi$, as can be verified by direct substitution in Eq. (15b).

Finally, if we replace the sinusoidal optical rotation with a saw-tooth optical rotation like that shown in Fig. 2(a) [and described by Eq. (5)], we will realize the magneto- or electro-optical analog of the rotating-compensator ellipsometer. ${ }^{12}$

${ }^{1}$ G. E. Sommargren, "Up/down frequency shifter for optical hetrodyne interferometry, "J. Opt. Soc. Am. 65, 960-961 (1975).

${ }^{2} \mathrm{~K}$. Serkowski, "Polarimeters for optical astronomy," in Planets, Stars and Nebulae Studied by Photopolarimetry, edited by T. Gehrels (University of Arizona Press, Tuscon, 1974). pp. 135-174.

${ }^{3}$ D. L. Coffeen, "Optical polarimeters in space," in Ref. 2, pp. 189-217.
${ }^{4}$ R. H. Muller, "Present status of automatic ellipsometers," Surf. Sci. 56, 19-36 (1976); also in Proceedings of the Third International Conference on Ellipsometry, edited by N. M. Bashara and R. M. A. Azzam (North-Holland, Amsterdam, 1976).

${ }^{5} \mathrm{D}$. E. Aspnes and P. S. Hauge, "Rotating-compensator/analyzer fixed analyzer ellipsometer: Analysis and comparison to other automatic ellipsometers, " J. Opt. Soc. Am. 66, 949-954 (1976).

${ }^{6} \mathrm{P}$. S. Hauge, "Survey of methods for the complete determination of a state of polarization," in Polarized Light: Instruments, Devices, and Applications, edited by W. L. Hyde and R. M. A. Azzam; also in Proc. Soc. Photo-Opt. Instrum., Eng., 88, pp. 3-10 (1976).

${ }^{7} \mathrm{~B}$. H. Billings, "The electro-optic effect in uniaxial crystals of the dihydrogen phosphate type," J. Opt. Soc. Am. 42 , 12-20 (1952).

${ }^{8} \mathrm{~J}$. C. Kemp, "Piezo-optical birefringence modulators: New use for a long-known effect," J. Opt. Soc. Am. 59, 950-954 (1969).

${ }^{9}$ R. M. A. Azzam, “Oscillating-analyzer ellipsometer," Rev. Sci. Instrum. 47, 624-628 (1976).

${ }^{10}$ See, for example, D. Clarke and J. F. Grainger, Polarized Light and Optical Mea surement (Pergamon, New York, 1971), p. 124 .

${ }^{11}$ Handbook of Mathematical Functions, edited by M. Abramowitz and J. A. Stegun (Dover, New York, 1965), p. 361.

${ }^{12} \mathrm{Z}$. Sekera, "Light scattering in the atmosphere and the polarization of sky light," J. Opt. Soc. Am. 47, 484-490 (1957); P. S. Hauge and F. H. Dill, "A rotating-compensator Fourier ellipsometer," Opt. Commun. 14, 431-434 (1975).

\title{
Photoemission studies of $\mathrm{LaF}_{3} \dagger$
}

\author{
W. Pong and C. S. Inouye \\ Department of Physics and Astronomy, University of Hawaii, Honolulu, Hawaii 96822
}

(Received 21 November 1977)

\begin{abstract}
Quantum-yield and photoelectron-energy-distribution measurements on evaporated $\mathrm{LaF}_{3}$ films have been made in the photon energy range $10-27 \mathrm{eV}$. Photoemission from the $F^{r} 2 p$ valence band indicates a bandwidth of $3.5 \pm 0.3 \mathrm{eV}$ and a photoelectric threshold of $11.7 \pm 0.3 \mathrm{eV}$. The photoelectron spectra exhibit features that can be identified as density-of-states structures of the valence and conduction bands. An attempt is made to relate these structures to the initial and final states of ultraviolet transitions.
\end{abstract}

\section{INTRODUCTION}

Recently, the optical properties of $\mathrm{LaF}_{3}$ have been studied by several investigators. The results of absorption measurements by Hunter et al. ${ }^{1}$ and Stephan et al. ${ }^{2}$ indicate a short wavelength transmittance limit of about $1250 \AA$. The onset of strong absorption for wavelengths below $1250 \AA$ is attributed to electronic transitions from the $\mathrm{F}^{-} 2 p$ valence band. The reflectivity spectra, measured in the $4-30 \mathrm{eV}$ range using synchrotron radiation, show structures that are identified as transitions from the $\mathrm{La}^{3+} 5 p$ and $\mathrm{F}^{-} 2 p$ states to the $5 d$ and $6 s$ states of the $\mathrm{La}^{3+}$ ions. ${ }^{3,4}$ The optical measurements provide useful information such as the allowed transition energies, but they do not give information on the location of the initial and final states. In order to give a more reliable interpretation of the optical data, it is necessary to study the density-of-states structure of $\mathrm{LaF}_{3}$.
In this paper we report the spectral quantum yield and the ultraviolet photoelectron spectra of $\mathrm{LaF}_{3}$. Threshold for photoemission from the $\mathrm{F}^{-} 2 p$ band is found to be $11.7 \pm 0.3$ $\mathrm{eV}$. The photoelectron energy distributions exhibit features that can be identified as density-of-states structures of the valence and conduction bands. An attempt is made to relate these structures to the initial and final states of ultraviolet transitions.

\section{EXPERIMENTAL}

The photoemission measurements were performed with the equipment previously described. ${ }^{5}$ Photons of energies up to $26.9 \mathrm{eV}$ were obtained by using a helium continuum source and a neon gas discharge. Thin $\mathrm{LaF}_{3}$ films were deposited on polished stainless steel substrates by evaporating $\mathrm{LaF}_{3}$ 